\title{
ON SECOND-CATEGORY SETS
}

\author{
PÉTER KOMJÁTH
}

(Communicated by Thomas J. Jech)

\begin{abstract}
The existence of a measurable cardinal is equiconsistent to the existence of a second category set not decomposable into the union of uncountable many disjoint second category sets.
\end{abstract}

It is easy to show that every second category set of reals can be decomposed into the disjoint union of infinitely many disjoint second category sets. Ulam [11] proved that under $\mathrm{CH}$ even uncountably many such sets can be found (see also [5], [9]). It is clear from saturated ideal theory that if there exists a set without such a decomposition, then there exists a model with a measurable cardinal. Also, the cardinality of the counter-example set is smaller than continuum but weakly Mahlo (see [8], [10]). In this note we show that these sets may exist.

Theorem. If the existence of a measurable cardinal is consistent, then so is the existence of a second category set in $R$ that is not the disjoint union uncountably many second category sets.

Proof. Let $V$ be a countable model with $\kappa$ a measurable cardinal and $I$ a $\kappa$-complete normal prime ideal on $\kappa$.

Let $P$ be the notion of forcing, adding $\kappa$ Cohen reals side-by-side with finite supports. We denote the $\alpha$ th Cohen real by $r_{\alpha}$. For every $A \in I$ in $V^{P}$, we let $Q(A)$ be the canonical notion of forcing, making $\left\{r_{\alpha}: \alpha \in A\right\}$ first category. This is defined as follows. Enumerate the diadic intervals as $\left\{I_{n}: n<\omega\right\} . q=\left(s, N, g, f_{0}, \ldots, f_{n}\right)$ is in $Q(A)$, if $s \in[A]^{<\omega}, N<\omega$, $f_{i}$ is a function defined on $\left\{I_{0}, \ldots, I_{N}\right\}$ with $f_{i}\left(I_{t}\right)$ a diadic subinterval of $I_{t}, g: s \rightarrow\{0,1 \ldots, n\}$, such that if $\alpha \in s, i=g(s)$, then $r_{\alpha} \notin f_{i}\left(I_{t}\right), t=$ $0,1, \ldots, N .\left(s^{\prime}, N^{\prime}, g^{\prime}, f_{0}^{\prime}, \ldots, f_{n^{\prime}}^{\prime}\right) \leq\left(s, N, g, f_{0}, \cdots, f_{n}\right)$ if $s^{\prime} \supseteq s, N^{\prime} \geq$ $N, g^{\prime} \supseteq g, f_{i}^{\prime} \supseteq f_{i}(i \leq n)$. It is well known that $Q(A)$ is ccc and as required. Clearly, $Q(A)$ is in $V\left[\left\{r_{\alpha}: \alpha \in A\right\}\right]$. By $\Delta$-system arguments, $Q=X\{Q(A): A \in I\}$ is ccc, and so $P * Q$ is also ccc.

Our aim is to show that in $V^{P, Q}, X=\left\{r_{\alpha}: \alpha<\kappa\right\}$ witnesses the theorem. Assume first that $p \Vdash$ “X $\left.X \bigcup \bigcup Y_{\xi}: \xi<\omega_{1}\right\}$ is a decomposition". We put

Received by the editors December 1, 1988.

1980 Mathematics Subject Classification (1985 Revision). Primary 28A05; Secondary 26A03, $03 \mathrm{E} 35,04 \mathrm{~A} 15$. 
$A_{\xi}=\left\{\alpha<\kappa: \exists p^{\prime} \leq p, p^{\prime} \Vdash\right.$ " $r_{\alpha} \in Y_{\xi}$ ” $\}$. These sets are defined in $V$. If an $A_{\xi}$ is in $I$, then forcing by $Q\left(A_{\xi}\right)$, and therefore forcing by $Q$, makes $Y_{\xi}$ first category. Otherwise, every $A_{\xi}$ is of measure one, i.e., $\kappa-A_{\xi} \in I$. Select an $\alpha \in \bigcap\left\{A_{\xi}: \xi<\omega_{1}\right\}$. For every $\xi<\omega_{1}$, there is a $p_{\xi} \leq p$, forcing $r_{\alpha} \in Y_{\xi}$. As $p$ forces so that the $Y_{\xi}-\mathrm{s}$ are disjoint, the $p_{\xi}-\mathrm{s}$ are pairwise incompatible, which contradicts the fact that $P * Q$ is ccc.

We now show that $X$ remains a set of second category even in $V^{P, Q}$. We may assume that $1 \| \frac{V}{P * Q}$ " $X$ is of first category", for otherwise we can find a model with an $X$ of second category, and we are done. By a well-known lemma of forcing theory, there are names $F_{0}, F_{1}, \ldots$ such that 1 forces the $F_{n}-\mathrm{s}$ to be closed, nowhere dense sets, which cover $X$. There are, in $V^{P}$, conditions $q_{i} \in Q$ that completely define the $F_{n}-\mathrm{s}$ by ccc. There are, in $V$, sets $A_{0}, A_{1}, \cdots \in I$ such that $\operatorname{supp}\left(q_{i}\right) \subseteq\left\{A_{0}, A_{1}, \ldots\right\}$, again by ccc. Select an $\alpha \in \kappa-\left(A_{0} \cup A_{1} \cup \cdots\right)$. If $Q^{\prime}=X\left\{Q\left(A_{i}\right): i<\omega\right\}, \cup F_{n}$ is in $V^{P, Q^{\prime}}$; so by absoluteness, $r_{\alpha} \in \bigcup F_{n}$ holds there. Let $H$ be $Q^{\prime}$-generic, choose a real $y$ which is not in $\bigcup F_{n}$ or in any of the first-category sets, coded in $V^{\prime}=V\left[\left\{r_{\beta}: \beta \neq \alpha\right\}, H\right]$. This is possible because our models are countable. Then, as is well known, $y$ is Cohen-generic over $V^{\prime}$ (see [4]). And in $V^{\prime}[y]$ the set $Y=X-\left\{r_{c}\right\} \cup\{y\}$ may have the same name as the one for $X$. U $F_{n}$ is also the same, but $y \notin \bigcup F_{n}$, a contradiction.

\section{REFERENCES}

1. D. H. Fremlin, Consequences of Martin's axiom, Cambridge Univ. Press, 1984.

2. G. L. Itzkowitz, Continuous measures, Baire category, and uniform continuity in topological groups, Pacific J. Math., 54 (1974), 115-125.

3. K. Kunen, Set theory, North-Holland, 1980.

4. __ Random and Cohen reals, Handbook of Set-Theoretic Topology (K. Kunen and J. E. Vaughan, eds.) North-Holland, 1984, pp. 887-911.

5. K. Kuratowski, Topology I, Academic Press, 1966.

6. D. A. Martin and R. M. Solovay, Internal Cohen extensions, Ann. Math. Logic 2 (1970), 143-178.

7. J. C. Morgan II, Baire category from an abstract viewpoint, Fund. Math. 94 (1977), 13-23.

8. K. L. Prikry, Changing measurable into accessible cardinals, Dissertationes Math. 68 (1970), 75-80.

9. W. Sierpiński, Hypothèse du continu, Chelsea Publ., New York, 1956.

10. R. M. Solovay, Real-ralued measurable cardinals, Axiomatic Set Theory, (D. S. Scott, ed.) Amer. Math. Soc., Providence, R.I., 1971, pp. 397-428.

11. S. Ulam, Üher gewisse Zerlegungen von Mengen, Fund. Math. 20 (1933), 221-223.

Department of Computer Science, R. Eötvös University, Budapest, Muzeum krt 6-8, 1088 HUNGARY 